\title{
As espécies brasileiras endêmicas de Campylopus Brid. (Bryophyta) estão ameaçadas? Uma análise usando modelagem para avaliar os seus estados de conservação
}

\author{
Marcela Inácio-Silva ${ }^{1,2}$, Dimas Marchi do Carmo $^{1}$ e Denilson Fernandes Peralta ${ }^{1}$ \\ Recebido: 13.02.2017; aceito: 27.06.2017
}

\begin{abstract}
Are the endemic brazilian species of Campylopus Brid. (Bryophyta) threatened? An analysis using modeling to assess their conservation status). Thirty of the 65 Neotropical species of Campylopus Brid. (Bryophyta, Leucobryaceae) occur widely distributed in Brazil. Therefore, identifying the environmental factors that affect the distribution of these species is essential to predict the future pattern of distribution and assess their conservation status of the genus. Using the DIVA-GIS software, distribution maps for species were elaborated from 118 occurrence points. The data were applied in a predictive modeling analysis of areas with the best climatic conditions for the establishment of the endemic Brazilian species of Campylopus (C. dichrostis, C. fragilis, C. gatro-alaris, C. gemmatus, C. julicaulis, C. subcuspidatus, C. thwaitesii, C. uleanus and $C$. widgrenii). The climatic variables were obtained from Worldclim (resolution $2.5 \mathrm{~min}$.). As a result, the current predictive modeling indicated the preference for the Cerrado and the Atlantic Rainforest. Considering a future scenario of global warming, the modeling suggests a decrease in the areas favorable for the occurrence of species of Campylopus.

Keywords: Conservation, DIVA-GIS, Geographic distribution models
\end{abstract}

RESUMO - (As espécies brasileiras endêmicas de Campylopus Brid. (Bryophyta) estão ameaçadas? Uma análise usando modelagem para avaliar os seus estados de conservação). Trinta 30 das 65 espécies neotropicais do gênero Campylopus Brid. (Bryophyta, Leucobryaceae) ocorrem amplamente distribuídas no Brasil. Portanto, identificar os fatores ambientais que afetam a distribuição dessas espécies é essencial para prever o futuro e o padrão de distribuição e avaliar o estado de conservação do gênero. Usando o software DIVA-GIS, os mapas de distribuição para as espécies foram elaborados a partir de 118 pontos de ocorrência. Os dados foram aplicados em uma análise de modelagem preditiva de áreas com as melhores condições climáticas para o estabelecimento das espécies endêmicas brasileiras analisadas de Campylopus (C. dichrostis, C. fragilis, C. gatro-alaris, C. gemmatus, $C$. julicaulis, C. subcuspidatus, $C$. thwaitesii, $C$. uleanus e $C$. widgrenii). As variáveis climáticas foram obtidas do Worldclim (resolução 2,5 min.). Como resultado, a modelagem preditiva atual indicou a preferência pelo Cerrado e pela Mata Atlântica. Considerando um cenário futuro de aquecimento global, a modelagem sugere uma diminuição nas áreas favoráveis à ocorrência de espécies de Campylopus.

Palavras-chave: Conservação, DIVA-GIS, Modelo de distribuição geográfica

\section{Introdução}

Briófitas são organismos sensíveis às mudanças ambientais, principalmente porque realizam trocas gasosas e de nutrientes por todo o gametófito, tornando-as importantes para detectar os efeitos biológicos das alterações climáticas, ambientais e ecológicas (Frahm \& Gradstein 1991).

No Brasil, a Mata Atlântica constitui a região fitogeográfica de maior riqueza desse grupo vegetal em termos de diversidade e endemismo, devido principalmente à sua amplitude altitudinal e latitudinal, abrangendo $88 \%$ das espécies brasileiras (Stehman et al. 2009, Gradstein \& Costa 2003), nesta formação vegetal são encontradas 1.337 espécies de briófitas (Costa \& Peralta 2015), das quais 386 (31\%) ocorrem no litoral norte do estado de São Paulo (Carmo et al. 2016). A Mata Atlântica era considerada uma das maiores florestas tropicais nas Américas, cobrindo cerca de 150 milhões de hectares ao longo de $3.300 \mathrm{~km}$ da costa brasileira. Apesar das severas restrições e leis aplicadas ao desmatamento, a taxa de perda de floresta ainda é muito alta (Metzger 2009), restando apenas $2 \%$ da cobertura original que está oficialmente sob

1. Programa de Pós-graduação em Biodiversidade Vegetal e Meio Ambiente, Núcleo de Pesquisa em Briologia, Instituto de Botânica, Caixa Postal 68041, 04045-972 São Paulo, SP, Brasil

2. Autor para correspondência: marcella.inacio@gmail.com 
proteção distribuída em 131 áreas protegidas (ICMBio 2016).

O Cerrado é o segundo maior domínio fitogeográfico do Brasil composto por um mosaico de vegetação, o qual apresenta as maiores taxas de conversão da cobertura natural em áreas antropizadas (Garcia 2014). A vegetação é frequentemente afetada pelo fogo, um fator que reduz as chances de desenvolvimento de uma brioflora diversificada, sendo a maior parte representada por musgos, e observam-se também antóceros e uma flora de hepáticas representada por Marchantiales e poucas epífitas tolerantes à seca (Gradstein et al. 2001). Atualmente, o Cerrado mantém $20 \%$ a $35 \%$ de sua vegetação natural, com apenas $2,25 \%$ oficialmente sob a proteção de 55 áreas de conservação ambiental (ICMBio 2016), e autores como Machado et al. (2004) concluíram que o Cerrado pode desaparecer completamente em 2030, por causa do grande avanço no processo de fragmentação deste bioma.

O estudo de Salazar et al. (2006), indicou a possível ocorrência de mudanças importantes nos biomas brasileiros para um futuro próximo (período de 2070 a 2100), em decorrência das mudanças climáticas. Segundo os autores, extensas áreas de Floresta Ombrófila Densa resultariam em Cerrado e grandes extensões de Caatinga em deserto (Shimizu 2007).

A distribuição geográfica das comunidades de vegetação e sua relação com o clima têm sido examinadas com modelos de distribuição de espécies. Estes modelos usam como hipótese central o clima como padrão dominante sobre a distribuição da vegetação (Salazar et al. 2006). O interesse por esse tipo de ferramenta é crescente, em razão da necessidade de informações rápidas e fundamentadas para direcionar ações de conservação e preservação ambiental, o que evita a perda acelerada da biodiversidade (Giannini 2012).

Segundo Siqueira \& Durigan (2007), algumas dessas técnicas envolvem uma modelagem baseada em análises ambientais, nas quais os algoritmos exploram por condições ambientais semelhantes àquelas onde as espécies foram encontradas, resultando em áreas potenciais onde as condições ambientais seriam propícias ao desenvolvimento dessas espécies. Dessa forma, os resultados obtidos com a modelagem corresponderiam a uma previsão, apoiando-se em dados do nicho da espécie, e projetando uma área que representa a distribuição potencial da espécie fundamentado esses resultados em camadas ambientais utilizadas na modelagem (Siqueira \& Peterson 2003).

As variáveis ambientais utilizadas para construir esses tipos de modelos normalmente estão em formato raster ou shapefile, e disponibilizadas em endereços eletrônicos que hospedam grandes bancos de dados produzidos por grupos de pesquisadores, como o Worldclim (Coelho et al. 2016, Hijmans et al. 2005).

Técnicas oriundas de modelagem de nicho ecológico foram utilizadas por Siqueira \& Peterson (2003), para desenvolver um primeiro passo no entendimento aos efeitos de mudanças climáticas na distribuição de espécies arbóreas do bioma Cerrado, através do cruzamento de informações entre os pontos de ocorrência com dados de coberturas ambientais resumindo as dimensões ecológicas para as espécies analisada. Atualmente, tais metodologias de modelagem são consideradas as mais apropriadas para referenciar áreas ambientais protegidas (Pressey \& Cowling 2001, Margules \& Sarkar 2007).

No Brasil, os primeiros trabalhos que tratam de conservação de briófitas com o uso de ferramentas de modelagem preditiva começaram a ser realizados a partir de 1999, através das análises nacionais e regionais de Costa \& Santos (2009). Uma pesquisa mais recente foi realizada por Silva et al. (2014), onde os autores analisaram dez espécies de briófitas (cinco hepáticas e cinco musgos), e desenvolvido um modelo de distribuição geográfica, a fim de compará-lo com a atual rede de proteção ambiental de Mata Atlântica. O resultado foi o reconhecimento de três centros de distribuição potencial: uma no nordeste, um central e outro no sudeste do Brasil. A área total de potencialidade de ocorrências das espécies foi sobreposta sob 83 Unidades de Conservação (apenas 27\%), menos do que o adequado para uma conservação eficiente das espécies de briófitas, sendo que, o corredor central foi à região com o maior índice de adequabilidade ambiental, mas com poucas Unidades de Conservação.

Anteriormente, Barros et al. (2012) realizaram uma modelagem de distribuição geográfica de cinco espécies de Metzgeria Raddi (hepática) com o objetivo de estabelecer padrões de distribuição e conservação em Mata Atlântica no Brasil. Conforme os resultados obtidos foram revelados diversas áreas altamente adequadas, embora não apresentasse registros das espécies. Segundo os autores, através da sobreposição dos mapas de distribuição das espécies e mapas de fragmentos florestais de Mata Atlântica, foi observado 
que $49 \%$ do potencial de presença de Metzgeria estão sob a cobertura florestal, enquanto o restante está atualmente protegido em áreas de conservação ambiental. Com isso, desempenhar expedições para coletas em áreas com alto potencial de ocorrência juntamente com o desenvolvimento de metodologias para conservação que podem ser propostas no domínio de Mata Atlântica (Faria 2013).

Sérgio et al. (2007) utilizaram o método de modelagem de nicho para quatro espécies de briófitas em Portugal, que constam na lista de espécies ameaçadas da IUCN [i.e., Antitrichia californica Sull. ex Lesq, Riccia sommieri Levier, Cryphaea heteromalla (Hedw.) D. Mohr e Racomitrium hespericum Sérgio, J. Muñoz \& Ochyra], para estimar a extensão da ocorrência das mesmas, utilizando registros de herbários. Estes autores concluíram que os modelos foram importantes para avaliar o grau de ocupação dessas espécies em território europeu, fornecendo subsídios para a proteção dessas espécies, pois indicaram áreas com alta probabilidade de ocorrência. Constatando que a utilização de coleções de herbários pode fornecer informações importantes e necessárias para implementação de procedimentos de áreas de conservação e proteção ambiental (Faria 2013).

Por fim, o uso dos modelos de distribuição geográfica para as espécies de Campylopus Brid. é importante pois das 165 espécies ocorrentes no mundo, 65 espécies apresentam distribuição neotropical e 30 ocorrem no Brasil (nove endêmicas) com ampla distribuição em todos os Estados e biomas brasileiros (Costa \& Peralta 2015). Esse elevado número de espécies torna o Brasil o segundo centro de diversidade neotropical deste gênero após os Andes (Frahm 1991). O padrão de distribuição das espécies pode mudar em respostas às alterações do clima ou podem se deslocar para áreas mais adequadas para a sua sobrevivência (Gatti 2013, Pearson \& Dawson 2003), causando um declínio na diversidade que resultará em uma mudança na estrutura, função dos ecossistemas, ciclagem de nutrientes e carbono (He et al. 2016).

Considerando que o conhecimento da biodiversidade no Brasil é um tema relevante e frequentemente relacionado como chave para o desenvolvimento científico nacional (Peralta 2005), este trabalho pretende apresentar uma modelagem preditiva de distribuição para nove espécies brasileiras endêmicas de Campylopus Brid., com o objetivo de estudar a sua distribuição atual nos domínios fitogeográficos brasileiro, Cerrado e Mata Atlântica e, desse modo, avaliar a conservação deste gênero em um cenário hipotético futuro das mudanças climáticas. Pretendemos indicar áreas geográficas que apresentam condições propícias para o desenvolvimento das espécies de Campylopus, e estabelecer novas rotas de deslocamento direcional e/ou redução ou aumentar suas áreas potenciais de ocorrência, baseado nas mudanças climáticas de temperatura e precipitação.

\section{Material e métodos}

Seleção de espécies e coleta de dados - Os critérios para a seleção das nove espécies-alvo foram: endemismo brasileiro, taxonomia estável, existência de revisões para o grupoe um bom número de espécimes disponíveis no Herbário Maria Eneyda P.K. Fidalgo (SP).

As nove espécies brasileiras endêmicas de Campylopus analisadas foram: C. dichrostis (Müll. Hal.) Paris, C. fragilis (Brid.) Bruch \& Schimp., C. gastro-alaris (Müll. Hal.) Paris, C. gemmatus (Müll. Hal.) Paris, C. julicaulis Broth., C. subcuspidatus (Hampe) A. Jaeger, C. thwaitesii (Mitt.) A. Jaeger, C. uleanus (Müll. Hal.) Broth. e C. widgrenii (Müll. Hal.) Mitt.

Os registros de ocorrência dos espécimes georreferenciadas foram obtidas no banco de dados do speciesLink (http://www.splink.org.br/), Frahm (1978, 1979, 1991, 1999 a,b) e aqueles depositados no Herbário SP tiveram a identificação confirmada, totalizando 118 pontos de ocorrência. Todos os registros foram compilados e organizados em uma planilha com todas as informações dos espécimes de herbário seguindo a metodologia apresentada por Maciel et al. (2010), tais como localização, coordenadas geográficas, nome do coletor, data e número da coleção, nome do determinante e tipo de vegetação onde a espécie foi encontrada. Os pontos de ocorrência obtidos foram consideradosa distribuição geográfica real das espécies e as análises realizadas para o grupo das espécies endêmicas, visaram avaliar o atual intervalo de distribuição no Cerrado e Mata Atlântica e fazer uma modelagem previsível dos habitats mais apropriados para ocorrência em um cenário de aquecimento global.

Área de estudo e variáveis ambientais - As coordenadas geográficas de amostras sem essa informação que eram imprescindíveis foram estimadas usando a ferramenta on-line GEOLOC do speciesLink porém, somente quando a descrição da localidade de coleta 
estava indicada. As análises complementares, de sobreposição dos dados de ocorrência das espécies de Campylopus com unidades de conservação, domínios fitogeográficos e áreas prioritárias para a conservação foram realizadas através do programa DIVA-GIS permitindo observar a potencial distribuição da espécie. Os mapas de Áreas de Proteção Ambiental no Brasil foram obtidos no site do Ministério do Meio Ambiente-MMA (MMA 2016).

Para desenvolver modelos de distribuição das áreas mais apropriadas para os 118 espécimes de Campylopus analisadas foram utilizamos camadas com dados climáticos atuais e futuras com projeções para o ano de 2050 pelo modelo CCM3 (Community Climate Model) (Duffy et al. 2003, Núñez-Colín \& Goytia-Jiménez 2009, Govindasamy et al. 2003).

As variáveis climáticas foram obtidas do projeto Worldclim (2016), resolução de 2,5 minutos, com aproximadamente $4,5 \mathrm{~km}^{2}$ de resolução espacial (Hijmans et al. 2005). Estas variáveis têm valores mensais de temperatura e precipitação, representando as médias da sazonalidade de temperatura e precipitação ao longo do ano as quais são amplamente utilizadas em estudos de modelagem de nicho ecológico (Hijmans et al. 2004, Hijmans et al. 2005). Este método é adequado para os estudos sobre os padrões de localização geográfica dos espécimes depositados em herbários (Ganeshaiah et al. 2003, Núñez-Colín e Goytia-Jiménez 2009).

\section{Resultados e Discussão}

Os resultados obtidos permitem orientar e aumentar a chance de sucesso de programas de recuperação de áreas perturbadas, além de contribuir para o aumento do conhecimento científico sobre características ecológicas e preferências de habitat das espécies de Campylopus Brid. estudadas.

A projeção da distribuição ótima atual das espécies endêmicas foi semelhante à distribuição conhecida da espécie, que abrange os Estados das regiões sul e sudeste do Brasil. Muito embora seja evidente que o clima é um fator limitante para a distribuição das espécies (Maciel et al. 2010), alguns táxons se destacam por serem encontrados ocorrendo desde as zonas mais úmidas até as mais secas, como Campylopus julicaulis e Campylopus uleanus (figura 1).

Campylopus julicaulis foi registrada em pontos altos da Floresta Amazônica, em áreas de bordas do Parque Nacional do Pico da Neblina e entorno da Reserva Extrativista Catuá-Ipixuna, ambas as áreas de proteção ambiental localizadas no Estado do Amazonas (figura 2). Para Campylopus uleanus, os registros foram no Parque Estadual do Morro do Chapéu na região do Piemonte da Chapada Diamantina, localizado na bacia hidrográfica do Rio Paraguaçu no Estado da Bahia (Inema 2017). A distribuição destas espécies pode ser explicada pela preferência por determinados tipos de hábitats que se repetem em distintas zonas fitogeográficas, mostrando-se adequadas para o estabelecimento dessas populações (Maciel et al. 2010). Essas áreas de altitudes elevadas são de interesse para o estudo da biodiversidade, não apenas por estarem mais protegidas da ação antropogênica, mas também por apresentarem ecossistemas bastante distintos das regiões que as circundam (Coelho et al. 2015).

A projeção para cenários presentes de regiões favoráveis para a distribuição de espécies de Campylopus, com base nos modelos gerados, revela áreas nos Estados de São Paulo, Rio de Janeiro e Minas Gerais (figura 3), destacando-se em regiões onde incluem clima tropical com temperatura geralmente elevadas ao longo do ano, com variações das estações seca e chuvosa, influenciada pela altitude ao norte, nordeste e noroeste do Estado de São Paulo (CEPAGRI 2017).

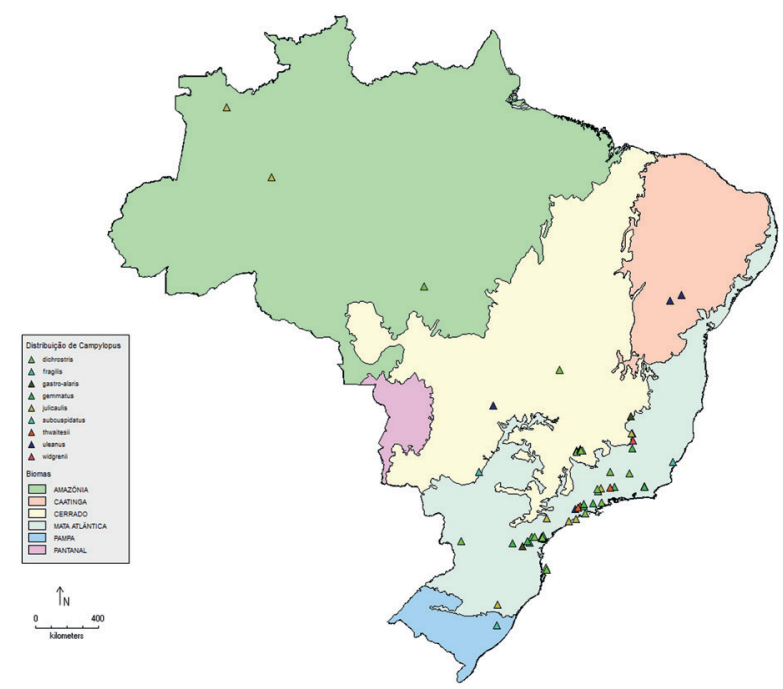

Figura 1. Modelo de distribuição atual das espécies de Campylopus $(C$. dichrostis, C. fragilis, C. gatro-alaris, C. gemmatus, $C$. julicaulis, $C$. subcuspidatus, C. thwaitesii, C. uleanus e C. widgrenii) ocorrentes nos biomas brasileiros. Dados obtidos através dos 118 pontos de ocorrência analisados com base em registros da coleção do Herbário SP.

Figure 1. Modeling of the current distribution of the species of Campylopus (C. dichrostis, C. fragilis, C. gatro-alaris, C. gemmatus, C. julicaulis, C. subcuspidatus, C. thwaitesii, C. uleanus and $C$. widgrenii) occurring in the Brazilian biomes. Data obtained from 118 occurrence points analyzed, based on records in the collections of the Herbarium SP. 


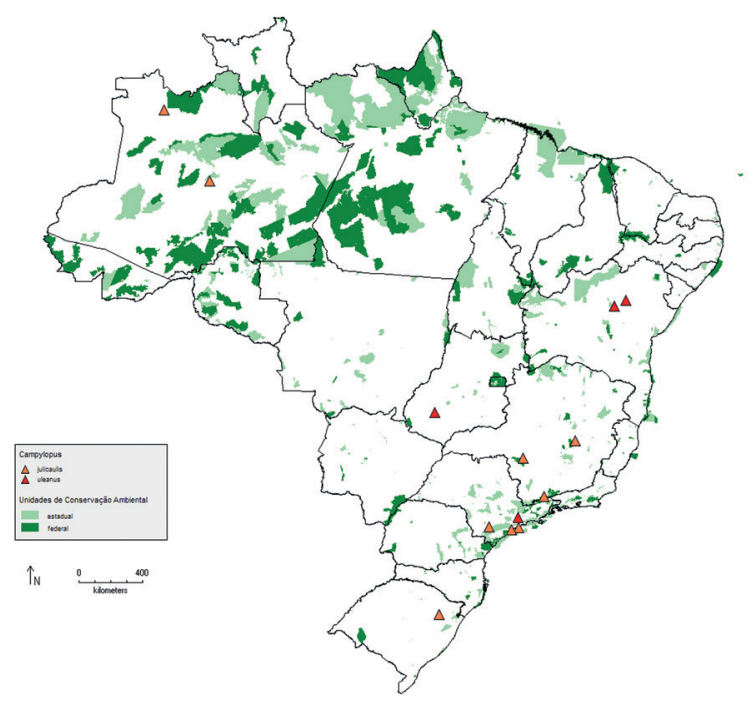

Figura 2. Áreas de Proteção Ambiental no Brasil de acordo com Ministério do Meio Ambiente (MMA).

Figure 2. Areas of Environmental Protection in Brazil, according to Ministry of the Environment (MMA).

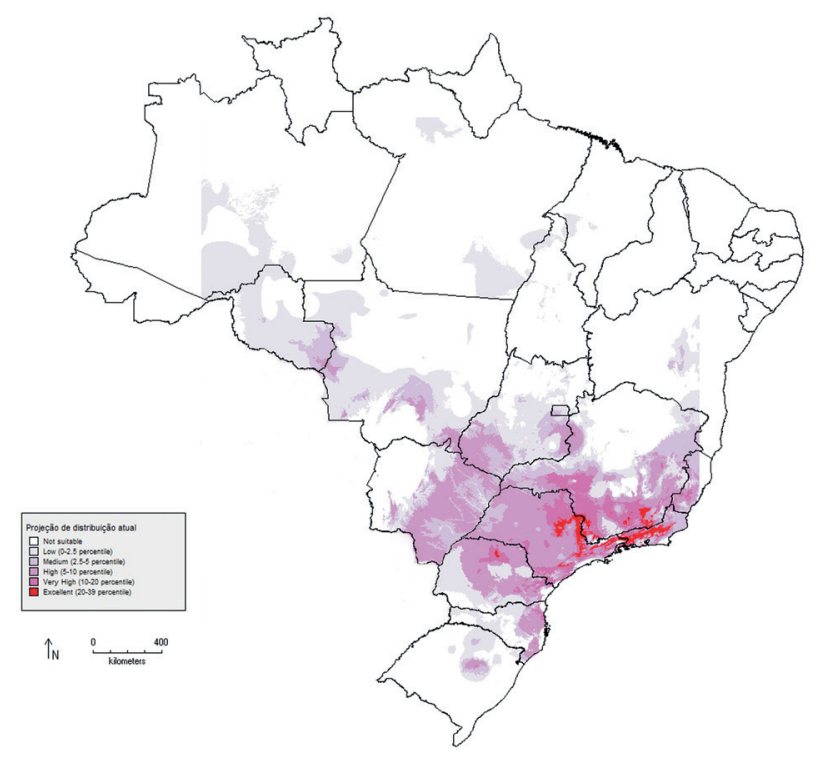

Figura 3. Modelo de distribuição mostrando o alcance atual para o estabelecimento de Campylopus ( $C$. dichrostis, $C$. fragilis, $C$. gatro-alaris, C. gemmatus, C. julicaulis, C. subcuspidatus, $C$. thwaitesii, C. uleanus e C. widgrenii) no Brasil. Dados obtidos através dos 118 pontos de ocorrência analisados e variáveis do WorldClim (resolução de 2,5 min).

Figure 3. Modeling of distribution showing the current potential extent for the establishment of Campylopus (C. dichrostis, C. fragilis, C. gatro-alaris, C. gemmatus, C. julicaulis, $C$. subcuspidatus, C. thwaitesii, C. uleanus and C. widgrenii) in Brazil. Data obtained from 118 occurrence points analyzed, and variables from WorldClim (resolution $2.5 \mathrm{~min}$.).
Um fator representativo é o clima no interior do Estado de São Paulo, na divisa noroeste e sul do Estado de Minas Gerais, onde estão localizados as regiões de Bragança Paulista, Mogi Mirim e São João da Boa Vista no Estado de São Paulo, a temperatura média nestas regiões é de $18,2{ }^{\circ} \mathrm{C}$ e uma pluviosidade média anual de $1.397 \mathrm{~mm}$. Em Poços de Caldas e São Sebastião do Paraíso cidades do Estado de Minas Gerais, o clima é quente e temperado e existe uma pluviosidade significativa ao longo do ano, mesmo o mês mais seco (Climate-Data 2016). Além da temperatura, os índices de precipitação foram importantes para Campylopus, que apresentou potencial de ocorrência em mais áreas do que as atualmente conhecidas.

Ao contrário de considerar somente cenários de predições atuais de distribuição, no presente estudo, avaliamos os efeitos das possíveis mudanças climáticas, a qual tem sido fator de risco de extinção de muitas espécies (Cassemiro et al. 2012), com base nos estudos de mudanças climáticas, do IPCC (Intergovernmental Panel on Climate Change), que reúne dados sobre a emissão de $\mathrm{CO}_{2}$ e de outros gases causadores do efeito estufa, através de modelos matemáticos, projetando os possíveis cenários futuros globais (especialmente para os próximos 50 anos (IPCC 1994, IPCC 1995 a, b)).

De acordo com o relatório do IPCC (2007), em um cenário mais otimista para os próximos 50 anos, estima-se um aumento de $0,5 \%$ /ano na concentração de $\mathrm{CO}_{2}$ na atmosfera e de cerca de $2{ }^{\circ} \mathrm{C}$ na temperatura média do planeta, entretanto, para um segundo cenário, mais pessimista, estima-se que um aumento de $1 \%$ /ano na concentração de $\mathrm{CO}_{2}$ na atmosfera tenha uma projeção na temperatura média de $4^{\circ} \mathrm{C}$. Há grandes evidências de que as mudanças climáticas são uma das maiores ameaças à diversidade de espécies (Loyola et al. 2008), e de acordo com Whittaker et al. (2001), fatores climáticos exercem papel fundamental sobre a distribuição das espécies em grandes escalas geográficas.

A modelagem de distribuição potencial das espécies revelou regiões com condições ótimas para as espécies de Campylopus, a partir da distribuição conhecida dessas espécies ecom base nos modelos gerados (figura 4), em regiões que incluem áreas prioritárias para conservação proposta pelo MMA (figura 2). É provável que em áreas previstas pelo modelo para sua ocorrência, mesmo que não existam 

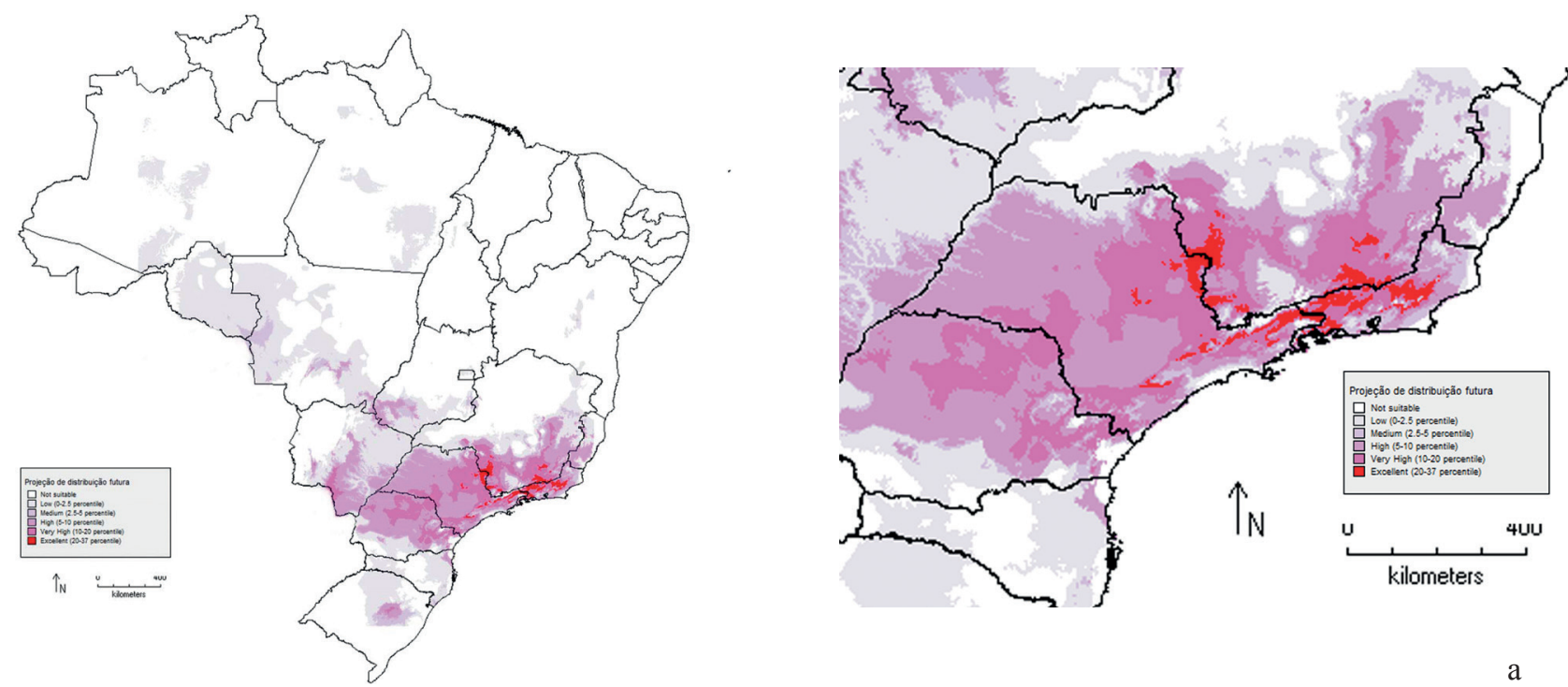

Figura 4. Modelo de distribuição mostrando previsões de alcance futuro para o estabelecimento Campylopus (C. dichrostis, C. fragilis, C. gatro-alaris, C. gemmatus, C. julicaulis, C. subcuspidatus, C. thwaitesii, C. uleanus e C. widgrenii) no Brasil. Dados obtidos através dos 118 pontos de ocorrência analisados e variáveis do WorldClim no Modelo CCM3. a. Detalhe da área de ocorrência futura mostrando a redução na área de potencial de distribuição para as espécies estudadas.

Figure 4. Modeling of distribution showing prediction of future extent for the establishment of Campylopus (C. dichrostis, C. fragilis, C. gatro-alaris, C. gemmatus, C. julicaulis, C. subcuspidatus, C. thwaitesii, C. uleanus and C. widgrenii) in Brazil. Data obtained from 118 occurrence points analyzed, and variables from WorldClim in the Model CCM3. a. Detail of the future extent area showing a decrease in the potential extent area for the studied species.

registros reais, as condições ambientais sejam propícias para desenvolvimento e manutenção de suas populações (Lopes et al. 2007). Por isso, é indicada aqui como áreas para empreender um maior esforço de coleta, a fim de localizar populações de Campylopus, como em áreas ao sul e sudoeste de Minas Gerais, a oeste do Estado do Rio de Janeiro, sul e nordeste e oeste do Estado de São Paulo, seguido pelo norte do Paraná e áreas do Rio Grande do Sul, onde possuem locais abertos e precipitações pluviométricas regulares, com clima temperado influenciado pelo domínio da Mata Atlântica e Pampa (MMA 2016).

Embora novas áreas tenham sido consideradas aptas, houve uma redução das áreas já conhecidas como adequadas para as ocorrências das espécies, principalmente ao norte e noroeste do Estado de São Paulo, nas regiões de Bragança Paulista e Mogi Mirim (figura 4a). Uma das possíveis causas destas mudanças de localização e tamanho das áreas reside no fato de que os cenários de mudanças climáticas apresentam diminuição da precipitação pluviométrica para as regiões subtropicais (IPCC 2007), além de um possível deslocamento destas para regiões mais ao norte do que as atualmente observadas no Estado de São Paulo. Devido ao aumento na temperatura global, espécies podem se deslocar e/ou sofrerem uma redução em suas áreas de ocorrência (Colombo 2007). Conforme Nobre et al. (2008), ecossistemas naturais como um todo não têm capacidade para migrar ou se adaptar à dimensão prevista das mudanças climáticas na escala de tempo, onde antes eram milhares de anos, se tornaram décadas.

Um efeito semelhante da migração foi encontrado por Nori et al. (2011), que relatou uma diminuição na adequação para habitats das rãs-touro (Rana catesbeiana Shaw (1802)), para uma distribuição potencial nos cenários futuros, com previsões para o ano de 2080 na América do Sul. Para esse modelo, as espécies de rãs-touro transferiram sua distribuição para áreas protegidas de Mata Atlântica, Floresta Andino-Patagônica, leste do Paraguai e noroeste da Bolívia, onde a adequabilidade ambiental era maior.

Colombo (2007) delineou áreas de distribuição geográfica futura para 38 espécies arbóreas típicas da Mata Atlântica. Este estudo mostrou um cenário pessimista com $50 \%$ de redução da ocorrência potencial de espécies até o ano 2050, mais uma possível migração para regiões sul das espécies atualmente existentes. Um cenário mais pessimista para o Cerrado foi descrito por Siqueira \& Peterson (2003), esses autores estudaram as consequências das mudanças climáticas na distribuição geográfica de 
162 espécies de árvores, mostrando que a diminuição e a perda de áreas de ocorrência para a maioria das espécies devem ser superiores a $50 \%$.

Este trabalho representa uma primeira contribuição para o estudo de espécies brasileiras endêmicas de briófitas e pode representar o ponto de partida para estudos adicionais sobre ecologia, biologia reprodutiva, barreiras, especiação e processos de extinção em Campylopus e outros gêneros. Torna-se imprescindível um conjunto de ações integradas que permitam não só a conservação dos remanescentes, mas que também assegurem, através de projetos de recomposição da vegetação, criação de Áreas de Preservação Permanente, com uma maior conectividade entre fragmentos hoje isolados (Colombo 2007).

Vellak et al. (2010) afirmou que a área de conservação para proteção das briófitas requer mais atenção, assim como a proteção e conservação de fanerógamas. A riqueza de espécies de briófitas e plantas vasculares está positivamente correlacionada, isto é, preservando a riqueza de uma comunidade vegetal, pode garantir a manutenção da riqueza de outras espécies (Ingerpuu et al. 2001).

A importância de considerar os dados obtidos apresentados neste estudo baseia-se na ocorrência atual de espécies para garantir a preservação e conservação efetiva para futuras gerações em possíveis áreas de maior diversidade no futuro (Colombo 2007). Além disso, esse estudo contribui com explorar as ligações entre a distribuição das espécies e fatores ambientais para preencher as lacunas existentes, identificar áreas que podem abrigar espécies raras e vulneráveis e fornecer uma base para estudos florísticos adicionais (Barros 2012). Por fim, com o modelo gerado é possível realizar inferências sobre distribuição geográfica da espécie, servindo em campanhas de campo, onde se tem o objetivo de localizar a espécie na natureza.

\section{Literatura citada}

Barros, F.S.M., Siqueira, M.F., Costa, D.P. 2012. Modeling the potential geographic distribution of five species of Metzgeria Raddi in Brazil, aiming at their conservation. The Bryologist 115: 341-349.

Carmo, D.M., Lima, J.S., Amélio, L.A., Peralta, D.F. 2016. Briófitas do Parque Estadual da Serra do Mar, Núcleo de Santa Virgínia, Estado de São Paulo, Brasil. Hoehnea 43: 265-287.

Cassemiro, F.A.S., Gouveia, S.F., \& Diniz-Filho, J.A.F. 2012. Distribuição de Rhinella granulosa: integrando envelopes bioclimáticos e respostas ecofisiológicas. Revista da Biologia 8: 38-44.
CEPAGRI - Centro de Pesquisas Meteorológicas e Climáticas Aplicadas à Agricultura. 2017. Disponível em http://www.cpa.unicamp.br/outras-informacoes/ clima-dos-municipios-paulistas.html (acesso em 30-I-2017).

Climate-Date. 2016. Dados climáticos para cidades mundiais. Disponível em https://pt.climate-data.org/ location/32843/ (acesso em 12-XII-2016).

Coelho, G.L.N., Carvalho, L.M.T., Gomide, L.R. 2016. Modelagem preditiva de distribuição de espécies pioneiras no Estado de Minas Gerais. Pesquisa Agropecuária Brasileira 51: 207-214.

Coelho, M.A.N., Costa, D.P., Martinelli, G., Moraes, M.A., Forzza, R.C. 2015. Expedições às montanhas da Amazônia $=$ Expeditions to the Mountains of Amazonia. Andrea Jakobsson Estúdio, Rio de Janeiro.

Colombo, A.F. 2007. Consequências potenciais das mudanças climáticas globais para espécies arbóreas de mata Atlântica. Dissertação de Mestrado, Universidade Estadual de Campinas, São Paulo.

Costa, D.P., Peralta, D.F. 2015. Bryophytes diversity in Brazil. Rodriguésia 66: 1063-1071.

Costa, D.P., Santos, N.D. 2009. Conservação de hepáticas na Mata Atlântica do sudeste do Brasil: uma análise regional no estado do Rio de Janeiro. Acta Botânica Brasílica 23: 913-922.

Duffy, P.B., Govindasamy, B., Iorio, J., Milovich, J., Sperber, K., Taylor, K.E., Wehner, M., Lamont, A., Thompson, S. 2003. High-resolution simulation of global climate, Part 1: presente. Climate Dynamics, 21: 371-390.

Faria, R.S. 2013. Distribuição dos gêneros Calymperes e Syrrhopodon (Calymperaceae, Bryophyta) em Floresta Atlântica Nordestina (Rio Grande do Norte a Sergipe): Fatores ambientais e história de vida. Dissertação de Mestrado, Universidade Federal de Pernambuco, Pernambuco.

Frahm, J.P. 1978. Übersicht der Campylopus-Arten der Anden. The Journal of the Hattori Botanical Laboratory 44: 483-524.

Frahm, J.P. 1979. Die Campylopus-ArtenBrasiliens. Revue Bryologique et Lichénologique 45: 127-178.

Frahm, J.P. 1981. Ergänzungenzur Campylopus- Flora Von Brasilien. Cryptogamie, Bryologie Lichénologie 2: 441-448.

Frahm, J.P. 1988. The Subantarctic and Southern Hemispheric species of Campylopus (Dicranaceae), with contributions to the origin and speciation of the genus. The Journal of the Hattori Botanical Laboratory 64: 367-387.

Frahm, J.P. 1991. Dicranaceae: Campylopodioideae, Paraleucobryoideae. Flora Neotropica 54: 1-238.

Frahm, J.P. 1996. Campylopus extinctus n. sp. (Musci, Dicranaceae), an apparently extinct species from Brazil. The Bryologist 99: 218-220. 
Frahm, J.P. 1999a. A type catalogue of Campylopodioideae and Paraleucobryoideae (Musci, Dicranaceae), Part II, Campylopus. Tropical Bryology 16: 17-102.

Frahm, J.P. 1999b. Taxonomische Notizenzur Gattung Campylopus XVIII. Tropical Bryology 17: 19-20.

Frahm, J.P., Gradstein, S.R. 1991. An altitudinal zonation of tropical rain forests using bryophytes, Journal of Bryology 18: 669-678.

Frahm, J.P., Vitt, D.H. 1978. A taxonomic study of Campylopus schimperi and C. subulatus (Bryopsida: Dicranaceae) in North America. Brittonia 30: 365- 372.

Ganeshaiah, K.N., Barve, N., Nath, N., Chandrashekara, K., Swamy, M., Shaanker, R.U. 2003. Predicting the potential geographical distribution of the sugarcane woolly aphid using GARP and DIVA-GIS. Current Science 85: 1526-1528.

Garcia, A.S. 2014. O Cerrado é o segundo maior bioma da América do Sul, o qual apresenta as maiores taxas de conversão da cobertura natural em áreas antropizadas. Dissertação de Mestrado, Universidade de São Paulo, São Paulo.

Gatti, A. 2013. Modelos de nicho, mudanças climáticas e a vulnerabilidade do clado Perissodactyla ao longo do tempo. Tese de Doutorado, Universidade Federal do Espírito Santo, Espírito Santo.

Giannini, T.C., Siqueira, M.F., Acosta, A.L., Barreto, F.C.C., Saraiva, A.M., Alves-Dos-Santos, I. 2012. Desafios atuais da modelagem preditiva de distribuição de espécies. Rodriguésia 63: 733-749.

Govindasamy, B., Duffy, P.B., Coquard, J. 2003. Highresolution simulations of global climate, part 2: effects of increased greenhouse cases. Climate Dynamics 21: 391-404.

Gradstein, S.R. \& Costa, D.P. 2003. The Hepaticae and Anthocerotae of Brazil. Mem. New York Botanical Garden 87: 1-336.

Gradstein, S.R., Churchill, S.P. \& Salazar-Allen, N. 2001. Guide to the bryophytes of Tropical America. Memoirs of the New York Botanical Garden 86: 1-577.

He, X., He, K.S. \&Hyvonen, J. 2016. Will bryophytes survive in a warming world? Perspectives in Plants Ecology. Evolution and Systematics 19: 49-60.

Hijmans, R.J., Cameron, S.E., Parra, J.L., Jones, P.G. \& Jarvis, A. 2005. Very high resolution interpolated climate surfaces for global land areas. International Journal of Climatology 25: 1965-1978.

Hijmans, R.J., Guarino, L., Bussink, C., Mathur, P., Cruz, M., Barrantes, I., Rojas, E. 2004. DIVA-GIS: sistema de información geográfica para El análisis de datos de distribución de especies. Version 4. InternationalPotato Center,Lima.

ICMBio - Instituto Chico Mendes. 2016. Unidades de Conservação nos biomas. Disponível em http:// www.icmbio.gov.br/portal/biodiversidade/unidadesde-conservacao/biomas-brasileiros.html (acesso em 20-III-2016).
INEMA - Instituto do meio Ambiente e Recursos Hídricos. 2017. Disponível em http://www.inema. ba.gov.br/gestao-2/unidades-de-conservacao/parqueestadual/parque-estadual-do-morro-do-chapeu/ (acesso em 30-I-2017).

Ingerpuu, N., Vellak, K., Kukk, T. \&Pärtel, M. 2001. Bryophyte and vascular plant species richness in boreo-nemoral moist forests and mires. Biodiversity and Conservation 10: 2153-2166.

International Panel on Climate Changes/IPCC GTI. 1995. Climate Change 1995 - The Science of Climate Change: Contribution of Working Group I to the Second Assessment Report of the Intergovernmental Panel on Climate Change. In: J.T. Houghton, L.G. MeiraFilho, B.A. Callander, N. Harris, A. Kattenberg e K. Maskell (eds.). Cambridge University Press, Cambridge.

International Panel on Climate Changes/IPCC GTII. 1995. Climate Change 1995 - Impacts, Adaptations and Mitigations of Climate Change: Scientific-Technical Analyses: Contribution of Working Group II to the Second Assessment Report of the Intergovernmental Panel on Climate Change. In: R.T. Watson, M.C. Zinyowera e R.H. Moss (eds.). Cambridge University Press, New York.

International Panel on Climate Changes/IPCC. 1994. Climate Change 1994: Radiative Forcing of Climate Change and an Evaluation of the IPCC IS92 Emission Scenarios. In: J.T. Houghton, L.G. Meira-Filho, J. Bruce, Hoesung Lee, B.A. Callander, E. Haites, N. Harris e K. Maskell (eds.). Cambridge University Press, Cambridge.

International Panel on Climate Changes/IPCC. 2007. Climate change 2007: Mitigation. Contribution of Working group III to the Fourth Assessment Report of the Intergovernmental Panel on Climate Change. In: B. Metz, O.R. Davidson, P.R. Bosch, R. Dave, L.A. Meyer (eds.). Cambridge University Press, Cambridge.

Lopes, T.S., Leite, V.R. \& Leite, G.R. 2007. Modelagem de Nicho Ecológico e Conservação de Dalbergia nigra, Espécie Ameaçada de Extinção. Revista Brasileira de Biociências 5: 438-440.

Loyola, R.D., Becker, C.G., Kubota, U., Haddad, C.F.B. \&Lewinsohn, T.M. 2008. Hung out to dry, choice of priority ecoregions for conserving threatened Neotropical anurans depends on life-history traits. PLoS ONE 3:e2120.

Machado, R.B., Ramos Neto, M.B., Pereira, P.G.P., Caldas, E.F., Gonçalves, D.A., Santos, N.S., Tabor, K. \& Steininger, M. 2004. Estimativas de perda da área do Cerrado brasileiro. Relatório técnico não publicado. Conservação Internacional, Brasília.

Maciel, J.R., Oliveira, R.C., Alves M. 2010. Aspectos da distribuição e conservação Paspalum L. (Poaceae) em Pernambuco. Hoehnea37: 801-812. 
Margules, C.R. \& Sarkar, S. 2007. Systematic conservation planning. Cambridge University Press, Cambridge.

Metzger, J.P. 2009. Conservation issues in the Brazilian Atlantic forest. Biological Conservation 142: 1138-1140.

MMA - Ministério do Meio Ambiente. 2016. Disponível em http://www.mma.gov.br/biomas/cerrado (acesso em 16-III-2016).

Nobre, C.A., Sampaio, G., Salazar, S. 2008. Cenários de mudanças climáticas para a América do Sul para o final do século 21. Parcerias Estratégicas, n. 27, pp. 19-41.

Nori, J., Urbina-Cardona, J. N., Loyola, R.D., Lescano, J.N., Leynaud, G.C. 2011. Climate Change and American Bullfrog Invasion: What Could We Expect in South America? PLoS ONE 6:e 25718.

Núñez-Colín, C.A. \& Goytia-Jiménez, M.A. 2009. Distribution and agroclimatic characterization of potential cultivation regions of physic nut in Mexico. Pesquisa Agropecuária Brasileira 44: 1078-1085.

Pearson, R.G. \& Dawson, T.P. 2003. Predicting the impacts of climate change on the distribution of species: are bioclimate envelope model suseful? Global Ecology and Biogeography 12: 361-371.

Peralta, D.F. 2005. Musgos (Bryophyta) do Parque Estadual da Ilha Anchieta (PEIA), São Paulo, Brasil. Dissertação de Mestrado, Instituto de Botânica, São Paulo.

Pressey, R.L. \& Cowling, R.M. 2001. Reserve selection algorithms and the real world. Conservation Biology 15: 275-277.

Salazar, L.F., Nobre, C.A., Oyama, M.D. 2006. Consequências das mudanças climáticas nos biomas da América do Sul. In: Congresso Brasileiro de Meteorologia, Florianópolis. Anais. [S.1]: Sociedade Brasileira de Meterologia. Disponível em http://www. cbmet.com/cbm-files/14-47c009e61 eb80cd2dff94205 51dd1a57.pdf (acesso em 02-II-2017).
Sérgio, C., Figueira, R., Draper, D., Menezes, R., Souza, A.J. 2007. Modelling bryophyte based on ecological information for extent of occurrence assessment. Biological Conservation 35: 341-351.

Shimizu, J.Y. 2007. Estratégia complementar para conservação de espécies florestais nativas: resgate e conservação de ecótipos ameaçados. Pesquisa Florestal Brasileira 54: 07-35.

Silva, M.P.P., Kamino, L.H.Y., Pôrto, K.C. 2014. Is the current network system of protected areas in the Atlantic Forest effective in conserving key species of bryophytes? Tropical Conservation Science 7: 61-74.

Siqueira, M.F., Durigan, G. 2007. Modelagem da distribuição geográfica de espécies lenhosas de cerrado no Estado de São Paulo. Revista Brasileira de Botânica 30: 233-243.

Siqueira, M.F., Peterson, A.T. 2003. Consequences of global climate change for geographic distributions of cerrado tree species. Biota Neotropica 3: 1-14.

Stehman, J., Forzza, R.C., Sobral, M. \& Kamino, L.H.Y. 2009. Gimnospermas e Angiospermas In: J. Stheman, R.C. Forzza, A. Salino, M. Sobral, D.P. Costa, L.H.Y. Kamino. Plantas da Floreta Atlântica,Rio de Janeiro, pp. 27-37.

Vellak, K., Ingerpuu, N., Vellak, A. \& Meelis, P. 2010. Vascular plant and bryophyte species representation in the protected areas network on the national scale. Biodiversity and Conservation 19: 1353-1364.

Whittaker, R., Willis, K.J. \& Field, R. 2001. Scale and species richness: towards a general, hierarchical theory of species diversity. Journal of Biogeography 28: 53-470. 